\title{
On Generalized Double Statistical Convergence of Order $\alpha$ in Intuitionistic Fuzzy Normed Spaces
}

\author{
Ekrem Savaş \\ Department of Mathematics, İstanbul Commerce University, Sutluce-İstanbul, 34672, Turkey; \\ ekremsavas@yahoo.com; Tel.: +90-505-2542198 \\ Academic Editor: Mehmet Pakdemirli \\ Received: 13 August 2015; Accepted: 12 June 2016; Published: 10 August 2016
}

\begin{abstract}
Our goal in this work is to introduce the notion $[V, \lambda](\mathcal{I})_{2}$-summability and ideal $\lambda$-double statistical convergence of order $\alpha$ with respect to the intuitionistic fuzzy norm $(\mu, v)$. We also make some observations about these spaces and prove some inclusion relations.
\end{abstract}

Keywords: ideal; filter; $\mathcal{I}$-double statistical convergence; $\mathcal{I}$-double statistical convergence order $\alpha$; $[V, \lambda]_{2}(\mathcal{I})$-summability; closed subspace

JEL: Primary 40G99

\section{Introduction}

Intuitionistic fuzzy set (IFNS) is one of the generalizations of fuzzy set theory [1]. Out of several higher-order fuzzy sets, IFNS, first introduced by Atanassov [2], have been found to be compatible to deal with vagueness. The conception of IFS can be viewed as an appropriate and alternative approach in cases where available information is not sufficient to define the impreciseness by the conventional fuzzy set theory. In fuzzy sets, only the degree of acceptance is considered. However, IFNS is characterized by a membership function and a non-membership function such that the sum of both values is less than one. Presently, intuitionistic fuzzy sets are being studied and used in different fields of science and engineering, e.g., population dynamics, chaos control, computer programming, nonlinear dynamical systems, fuzzy physics, fuzzy topology, etc. The concept of an intuitionistic fuzzy metric space was introduced by Park [3]. Furthermore, Saadati and Park [4] gave the notion of an intuitionistic fuzzy normed space.

In recent years, fuzzy topology has proven to be a very useful tool to deal with such situations where the use of classical theories breaks down. The most popular application of fuzzy topology in quantum particle physics aries in the string and $\varepsilon^{\infty}$-theory of El-Nashie [5] who presented the relation of fuzzy Kähler interpolation of $\varepsilon^{\infty}$ to the recent work on cosmo-topology. In [6], E. Savas introduced $\lambda$-double sequence spaces of fuzzy real numbers defined by Orlicz function.

The term "statistical convergence" was first defined by Fast [7] and Schoenberg [8] independently, which is a generalization of the concept of ordinary convergence. Statistical convergence appears in many fields such as in the theory of Fourier analysis, ergodic theory and number theory. Later on it was further investigated from the sequence space point of view and linked with summability theory by Fridy [9], Salát [10], Cakalli [11], Di Maio and Kocinac [12] and many others.

Definition 1. Let $K$ be a subset of $\mathbb{N}$, the set of natural numbers. Then the asymptotic density of $K$ denoted by $\delta(K),($ see, [13] is defined as

$$
\delta(K)=\lim _{n} \frac{1}{n}|\{k \leq n: k \in K\}|
$$

where the vertical bars denote the cardinality of the enclosed set. 
Definition 2. A number sequence $x=\left(x_{k}\right)$ is said to be statistically convergent to the number $L$ if for each $\varepsilon>0$, the set $K(\varepsilon)=\left\{k \leq n:\left|x_{k}-L\right|>\varepsilon\right\}$ has asymptotic density zero, i.e.,

$$
\lim _{n} \frac{1}{n}\left|\left\{k \leq n:\left|x_{k}-L\right|>\varepsilon\right\}\right|=0
$$

In this case we write st $-\lim x=L($ see, $[7,9])$.

Note that every convergent sequence is statistically convergent to the same limit, but the converse need not be true.

On the other hand, Mursaleen [14] introduced the concept of $\mathcal{I}_{\lambda}$-statistical convergence as a generalization of the statistical convergence and studied its relation to statistical convergence, Cesàro summability and strong $(V, \lambda)$-summability. In [15] we used ideals to introduce the concept of $\mathcal{I}_{\lambda}$-statistical convergence and study their some properties.

Furthermore, in $[16,17]$ a different direction was given to the study of these important summability methods where the notions of statistical convergence of order $\alpha$ and $\lambda$-statistical convergence of order $\alpha$ were introduced and studied.

In [18], P. Kostyrko et al. introduced and investigated $\mathcal{I}$-convergence of sequences in a metric space which is an interesting generalization of statistical convergence and studied some properties of such convergence. Subsequently, more investigations and more applications of ideals were introduced and studied in different directions, for instance, see [15,19-25].

Also, in [26] Mohiuddine and Lohani introduced the notion of the generalized statistical convergence in intuitionistic fuzzy normed spaces. Some works related to the convergence of sequences in several normed linear spaces in a fuzzy setting can be found in [26,27].

Quite recently, $\mathcal{I}$-double statistical convergence has been established as a better study than double statistical convergence. It is very interesting that some results on sequences, series and summability can be proved by replacing the double statistical convergence by $\mathcal{I}$-double statistical convergence. Also, it should be noted that the results of $\mathcal{I}_{\lambda}$-double statistical convergence in an intuitionistic fuzzy normed linear space happen to be stronger than those proved for $\lambda$-double statistical convergence in an intuitionistic fuzzy normed linear space.

In this paper, we intend to use ideals to introduce the concept of $\mathcal{I}_{\lambda}$-double statistical convergence of order $\alpha$ with respect to the intuitionistic fuzzy normed space $(\mu, v)$, and study some of its consequences.

It should be noted that throughout the paper, $\mathbb{N}$ will denote the set of all natural numbers.

The following definitions and notions will be needed in the sequel.

Definition 3. A triangular norm (t-norm) is a continuous mapping $*:[0,1] \times[0,1] \rightarrow[0,1]$ such that $(S, *)$ is an abelian monoid with unit one and $c * d \leq a * b$ if $c \leq a$ and $d \leq b$ for all $a, b, c, d \in[0,1]$ [28].

Definition 4. A binary operation $\diamond:[0,1] \times[0,1] \rightarrow[0,1]$ is said to be a continuous $t$-conorm if it satisfies the following conditions [28]:

(i) $\diamond$ is associate and commutative,

(ii) $\diamond$ is continuous,

(iii) $a \diamond 0=$ a for all $a \in[0,1]$,

(iv) $a \diamond b \leq c \diamond d$ whenever $a \leq c$ and $b \leq d$ for each $a, b, c, d \in[0,1]$.

For example, we can give $a * b=a b, a * b=\min \{a, b\}, a \diamond b=\min \{a+b, 1\}$ and $a \diamond b=\max \{a, b\}$ for all $a, b \in[0,1]$.

Using the continuous $t$-norm and $t$-conorm, Saadati and Park [4] has recently introduced the concept of intuitionistic fuzzy normed space as follows.

Definition 5. The five-tuple $(X, \mu, v, *, \diamond)$ is said to be an intuitionistic fuzzy normed space (for short, IFNS) if $X$ is a vector space, $*$ is a continuous $t$-norm, $\diamond$ is a continuous $t$-conorm, and $\mu$, $v$ are fuzzy sets on $X \times(0, \infty)$ satisfying the following conditions for every $x, y \in X$, and $s, t>0$ [4]: 
(a) $\mu(x, t)+v(x, t) \leq 1$,

(b) $\mu(x, t)>0$,

(c) $\mu(x, t)=1$ if and only if $x=0$,

(d) $\mu(\alpha x, t)=\mu\left(x, \frac{t}{|\alpha|}\right)$ for each $\alpha \neq 0$,

(e) $\mu(x, t) * \mu(y, s) \leq \mu(x+y, t+s)$,

(f) $\mu(x,):.(0, \infty) \rightarrow[0,1]$ is continuous,

(g) $\lim _{t \rightarrow \infty} \mu(x, t)=1$ and $\lim _{t \rightarrow 0} \mu(x, t)=0$,

(h) $v(x, t)<1$,

(i) $v(x, t)=0$ if and only if $x=0$,

(j) $v(\alpha x, t)=\mu\left(x, \frac{t}{|\alpha|}\right)$ for each $\alpha \neq 0$,

(k) $v(x, t) \diamond v(y, s) \geq v(x+y, t+s)$,

(l) $v(x,):.(0, \infty) \rightarrow[0,1]$ is continuous,

(m) $\lim _{t \rightarrow \infty} v(x, t)=0$ and $\lim _{t \rightarrow 0} v(x, t)=1$.

In this case $(\mu, v)$ is called an intuitionistic fuzzy norm. As a standard example, we can give the following:

Let $(X,\|\|$.$) be a normed space, and let a * b=a b$ and $a \diamond b=\min \{a+b, 1\}$ for all $a, b \in[0,1]$. For all $x \in X$ and every $t>0$, consider

$$
\mu(x, t)=\frac{t}{t+\|x\|} \text { and } v(x, t)=\frac{\|x\|}{t+\|x\|}
$$

Then observe that $(X, \mu, v, *, \diamond)$ is an intuitionistic fuzzy normed space.

We also recall that the concept of double convergence in an intuitionistic fuzzy normed space is studied in [29].

Definition 6. Let $(X, \mu, v, *, \diamond)$ be an IFNS. Then, a sequence $x=\left(x_{k, l}\right)$ is said to be convergent to $L$ with respect to the intuitionistic fuzzy norm $(\mu, v)$ if, for every $\varepsilon>0$ and $t>0$, there exists $\left(k_{0}, l_{0}\right) \in \mathbb{N} \times \mathbb{N}$ such that $\mu\left(x_{k, l}-L, t\right)>1-\varepsilon$ and $v\left(x_{k, l}-L, t\right)<\varepsilon$ for all $k \geq k_{0}$ and $l \geq l_{0}$. It is denoted by $(\mu, v)_{2}-\lim x=$ L or $x_{k, l} \stackrel{(\mu, v)_{2}}{\rightarrow}$ Las $k, l \rightarrow \infty[29]$.

\section{2. $\mathcal{I}_{\lambda}$-Double Statistical Convergence on IFNS}

In this section we deal with the relation between these two new methods as also the relation between $\mathcal{I}_{\lambda}$-double statistical convergence and $\mathcal{I}$-double statistical convergence in an intuitionistic fuzzy normed space $(\mu, v)$. Before proceeding further, we should recall some notation on the $\mathcal{I}$-double statistical convergence and ideal convergence.

The idea of $\lambda$-statistical convergence of single and double sequences of fuzzy numbers has been studied by Savas $[6,30]$ respectively .

Statistical convergence of double sequences $x=\left(x_{k l}\right)$ has been defined and studied by Karakus, S., Demirci, K. and Duman, O. [31]; and for fuzzy numbers by Savas and Mursaleen [32].

Now, it would be helpful to give some definitions.

Let $K \subseteq \mathbb{N} \times \mathbb{N}$ be a two-dimensional set of positive integers and let $K(m, n)$ be the numbers of $(k, l)$ in $K$ such that $k \leq m$ and $l \leq n$. Then the two-dimensional analogue of natural density can be defined as follows [33].

The lower asymptotic density of the set $K \subseteq \mathbb{N} \times \mathbb{N}$ is defined as

$$
\underline{\delta_{2}}(K)=\liminf _{m, n} \frac{K(m, n)}{m n}
$$

In case the sequence $(K(m, n) / m n)$ has a limit then we say that $K$ has a double natural density and is defined as

$$
\lim _{m, n} \frac{K(m, n)}{m n}=\delta_{2}(K)
$$


Definition 7. A real double sequence $x=\left(x_{k, l}\right)$ is said to be statistically convergent to the number $\ell$ if for each $\varepsilon>0$, the set [33]

$$
\left\{(k, l), k \leq m \text { and } l \leq n:\left|x_{k, l}-\ell\right| \geq \varepsilon\right\}
$$

has double natural density zero. In this case we write $s_{2}-\lim _{k, l} x_{k, l}=\ell$.

We define the concept of double $\lambda$-density:

Let $\lambda=\left(\lambda_{m}\right)$ and $\mu=\left(\mu_{n}\right)$ be two non-decreasing sequences of positive real numbers both of which tends to $\infty$ as $m$ and $n$ approach $\infty$, respectively. Also let $\lambda_{m+1} \leq \lambda_{m}+1, \lambda_{1}=0$ and $\mu_{n+1} \leq \mu_{n}+1, \mu_{1}=0$. The collection of such sequence $(\lambda, \mu)$ will be denoted by $\Delta$.

Let $K \subseteq \mathbb{N} \times \mathbb{N}$. The number

$$
\delta_{\lambda}(K)=\lim _{m n} \frac{1}{\lambda_{m n}}\left|\left\{k \in I_{n}, l \in J_{m}:(k, l) \in K\right\}\right|
$$

where $I_{m}=\left[m-\lambda_{m}+1, m\right]$ and $J_{n}=\left[n-\mu_{n}+1, n\right]$ and $\lambda_{m n}=\lambda_{m} \mu_{n}$, is said to be the $\lambda$-density of $K$, provided the limit exists.

The family $\mathcal{I} \subset 2^{Y}$ of subsets a nonempty set $Y$ is said to be an ideal in $Y$ if (i) $\varnothing \notin \mathcal{I}$; (ii) $A, B \in \mathcal{I}$ imply $A \cup B \in \mathcal{I}$; (iii) $A \in \mathcal{I}, B \subset A$ imply $B \in \mathcal{I}$, while an admissible ideal $\mathcal{I}$ of $Y$ further satisfies $\{x\} \in \mathcal{I}$ for each $x \in Y$. If $\mathcal{I}$ is an ideal in $Y$ then the collection $F(\mathcal{I})=\left\{M \subset Y: M^{c} \in \mathcal{I}\right\}$ forms a filter in $Y$ which is called the filter associated with $\mathcal{I}$. Let $\mathcal{I} \subset 2^{\mathbb{N}}$ be a nontrivial ideal in $\mathbb{N}$. Then a sequence $\left\{x_{n}\right\}_{n \in \mathbb{N}}$ in $X$ is said to be $\mathcal{I}$-convergent to $x \in X$, if for each $\varepsilon>0$ the set $A(\varepsilon)=\left\{n \in \mathbb{N}:\left\|x_{n}-x\right\| \geq \varepsilon\right\}$ belongs to $\mathcal{I}$ (see [18]).

Throughout $\mathcal{I}$ will stand for a proper admissible ideal in $\mathbb{N} \times \mathbb{N}$.

Definition 8. A sequence $x=\left(x_{k, l}\right)$ is said to be $\mathcal{I}$-double statistically convergent of order $\alpha$ to $L$ or $S_{2}^{\alpha}(\mathcal{I})$-convergent to $L$, where $0<\alpha \leq 1$, if for each $\varepsilon>0$ and $\delta>0$ [24]

$$
\left\{(m, n) \in \mathbb{N} \times \mathbb{N}: \frac{1}{(m n)^{\alpha}} \mid\left\{k \leq m \text { and } l \leq n:\left|x_{k, l}-L\right| \geq \varepsilon\right\} \mid \geq \delta\right\} \in \mathcal{I}
$$

or equivalently if for each $\varepsilon>0$

$$
\delta_{\mathcal{I}}^{2}(A(\varepsilon))=\mathcal{I}-\lim \delta_{m n}(A(\varepsilon))=0
$$

where $A(\varepsilon)=\left\{k \leq m\right.$ and $\left.l \leq n:\left\|x_{k, l}-L\right\| \geq \varepsilon\right\}$ and $\delta_{m n}(A(\varepsilon))=\frac{|A(\varepsilon)|}{(m n)^{\alpha}}$.

In this case we write $x_{k, l} \rightarrow L\left(S_{2}^{\alpha}(\mathcal{I})\right)$. The class of all $\mathcal{I}$ - double statistically convergent of order $\alpha$ sequences will be denoted by simply $S_{2}^{\alpha}(\mathcal{I})$.

Remark 1. For $\mathcal{I}=\mathcal{I}_{\text {fin }}=\{A \subset \mathbb{N} \times \mathbb{N}: A$ is a finite $\}, S_{2}^{\alpha}(\mathcal{I})$-convergence coincides with double statistical convergence of order $\alpha$. For an arbitrary ideal $\mathcal{I}$ and for $\alpha=1$ it coincides with $\mathcal{I}$-double statistical convergence [24]. When $\mathcal{I}=\mathcal{I}_{\text {fin }}$ and $\alpha=1$ it becomes only double statistical convergence [33].

Definition 9. Let $\mathcal{I}$ be a nontrivial admissible ideal in $\mathbb{N} \times \mathbb{N}$. Let $(X, \mu, v, *, \diamond)$ be an IFNS. Then, a sequence $x=\left(x_{k, l}\right)$ is said to be $\mathcal{I}$-double statistically convergent of order $\alpha$ to $L \in X$, where $0<\alpha \leq 1$, with respect to the intuitionistic fuzzy normed space $(\mu, v)$, if for every $\varepsilon>0$, and every $\delta>0$ and $t>0$,

$$
\left\{(m, n) \in \mathbb{N} \times \mathbb{N}: \frac{1}{(m n)^{\alpha}} \mid\left\{k \leq m \text { and } l \leq n: \mu\left(x_{k, l}-L, t\right) \leq 1-\varepsilon \text { or } v\left(x_{k, l}-L, t\right) \geq \varepsilon\right\} \mid \geq \delta\right\} \in \mathcal{I}
$$

In this case we write $x_{k, l} \stackrel{(\mu, v)}{\rightarrow} L\left(S_{2}^{\alpha}(\mathcal{I})^{(\mu, v)}\right)$.

Remark 2. For $\mathcal{I}=\mathcal{I}_{\text {fin }}, S_{2}^{\alpha}(\mathcal{I})$-convergence coincides with statistical convergence of order $\alpha$, with respect to the intuitionistic fuzzy normed space $(\mu, v)$. For an arbitrary ideal $\mathcal{I}$ and for $\alpha=1$ it coincides with $\mathcal{I}$-double 
statistical convergence, with respect to the intuitionistic fuzzy normed space $(\mu, v),\left(\right.$ see, [27]). When $\mathcal{I}=\mathcal{I}_{\text {fin }}$ and $\alpha=1$ it becomes only double statistical convergence with respect to the intuitionistic fuzzy normed space $(\mu, v),[34]$.

We write the generalized double de la Valée-Poussin mean by

$$
t_{m, n}(x)=\frac{1}{\lambda_{m} \mu_{n}} \sum_{k \in I_{m}, l \in J_{n}} x_{k, l}
$$

A sequence $x=\left(x_{k, l}\right)$ is said to be $[V, \lambda]_{2}(\mathcal{I})$-summable to a number $L \in X$, if

$$
\mathcal{I}-\operatorname{limt}_{m, n}(x)=L
$$

i.e., for any $\delta>0$,

$$
\left\{(m, n) \in \mathbf{N} \times \mathbf{N}:\left|t_{m, n}(x)-L\right| \geq \delta\right\} \in \mathcal{I}
$$

Throughout this paper we shall denote $\lambda_{m} \mu_{n}$ by $\lambda_{m, n}$ and $\left(k \in I_{m}, l \in J_{n}\right)$ by $(k, l) \in I_{m n}$. We are now ready to obtain our main results.

Definition 10. Let $(X, \mu, v, *, \diamond)$ be an IFNS. We say that a sequence $x=\left(x_{k, l}\right)$ is said to be $[V, \lambda]_{2}(\mathcal{I})$-summable of order $\alpha$ to $L \in X$ with respect to the intuitionistic fuzzy normed space $(\mu, v)$, if for any $\delta>0$ and $t>0$,

$$
\left\{(m, n) \in \mathbb{N} \times \mathbb{N}: \mu\left(t_{m n}(x)-L, t\right) \leq 1-\delta \text { or } v\left(t_{m n}(x)-L, t\right) \geq \delta\right\} \in \mathcal{I}
$$

In this case we write $[V, \lambda]_{2}^{\alpha}(\mathcal{I})^{(\mu, v)}-\lim x=L$.

Definition 11. Let $(X, \mu, v, *, \diamond)$ be an IFNS. A sequence $x=\left(x_{k, l}\right)$ is said to be $\mathcal{I}_{\lambda}$-double statistically convergent of order $\alpha$ or $S_{\lambda}^{\alpha}(\mathcal{I})_{2}$-convergent to $L \in X$ with respect to the intuitionistic fuzzy normed space $(\mu, v)$, if for every $\varepsilon>0, \delta>0$ and $t>0$,

$$
\begin{aligned}
& \left\{(m, n) \in \mathbb{N} \times \mathbb{N}: \frac{1}{\lambda_{m n}^{\alpha}} \mid\left\{(k, l) \in I_{m n}: \mu\left(x_{k, l}-L, t\right) \leq 1-\varepsilon \text { or } v\left(x_{k, l}-L, t\right) \geq \varepsilon\right\} \mid \geq \delta\right\} \in \mathcal{I} \\
& \text { In this case we write } S_{\lambda}^{\alpha}(\mathcal{I})_{2}^{(\mu, v)}-\lim x=L \text { or } x_{k, l} \rightarrow L\left(S_{\lambda}^{\alpha}(\mathcal{I})_{2}^{(\mu, v)}\right) .
\end{aligned}
$$

Remark 3. For $\mathcal{I}=\mathcal{I}_{\text {fin }}, S_{\lambda}^{\alpha}(\mathcal{I})_{2}$--convergence with respect to the intuitionistic fuzzy normed space $(\mu, v)$, again coincides with $\lambda$-double statistical convergence of order $\alpha$, with respect to the intuitionistic fuzzy normed space $(\mu, v)$. For an arbitrary ideal $\mathcal{I}$ and for $\alpha=1$ it coincides with $\mathcal{I}_{\geq}$-double statistical convergence with respect to the intuitionistic fuzzy normed space $(\mu, v)$. Finally, for $\mathcal{I}=\mathcal{I}_{\text {fin }}$ and $\alpha=1$ it becomes $\lambda$-double statistical convergence with respect to the intuitionistic fuzzy normed space $(\mu, v)$ [29]. Also note that taking $\lambda_{m n}=m n$ we get Definition 9 from Definition 11.

We denote by $S_{\lambda}^{\alpha}(\mathcal{I})_{2}^{(\mu, v)}$ and $[V, \lambda]_{2}^{\alpha}(\mathcal{I})^{(\mu, v)}$ the collections of all $S_{\lambda}(\mathcal{I})_{2}$-convergent of order $\alpha$ and $[V, \lambda](\mathcal{I})_{2}$-convergent of order $\alpha$ sequences respectively.

We now have

Theorem 1. Let $(X, \mu, v, *, \diamond)$ be an IFNS. Let $\lambda=\left(\lambda_{m n}\right) \in \Delta$. Then $x_{k, l} \rightarrow L\left([V, \lambda]_{2}^{\alpha}(\mathcal{I})^{(\mu, v)}\right) \Rightarrow$ $x_{k, l} \rightarrow L\left(S_{\lambda}^{\alpha}(\mathcal{I})_{2}^{(\mu, v)}\right)$. 
Proof. By hypothesis, for every $\varepsilon>0, \delta>0$ and $t>0$, let $x_{k, l} \rightarrow L[V, \lambda]_{2}^{\alpha}(I)^{(\mu, v)}$. We have

$$
\begin{array}{ll}
\geq & \sum_{(k, l) \in I_{m n}}\left(\mu\left(x_{k, l}-L, t\right) \leq 1-\varepsilon \text { or } v\left(x_{k, l}-L, t\right) \geq \varepsilon\right) \\
& \sum_{\substack{(k, l) \in I_{m n} \& \mu\left(x_{k, l}-L, t\right)<1-\varepsilon \\
\text { or } v\left(x_{k, l}-L, t\right)>\varepsilon}}\left(\mu\left(x_{k, l}-L, t\right) \leq 1-\varepsilon \text { or } v\left(x_{k, l}-L, t\right) \geq \varepsilon\right) \\
\geq & \varepsilon \mid\left\{(k, l) \in I_{m n}: \mu\left(x_{k, l}-L, t\right) \leq 1-\varepsilon \text { or } v\left(x_{k, l}-L, t\right) \geq \varepsilon\right\} \mid
\end{array}
$$

Then observe that

$$
\begin{aligned}
& \frac{1}{\lambda_{m n}^{\alpha}} \mid\left\{(k, l) \in I_{m n}: \mu\left(x_{k, l}-L, t\right) \leq 1-\varepsilon \text { or } v\left(x_{k, l}-L, t\right) \geq \varepsilon\right\} \mid \geq \delta \\
\Rightarrow & \frac{1}{\lambda_{m n}^{\alpha}} \sum_{(k, l) \in I_{m n}} \mu\left(x_{k, l}-L, t\right) \leq(1-\varepsilon) \delta \text { or } \frac{1}{\lambda_{m n}^{\alpha}} \sum_{(k, l) \in I_{m n}} v\left(x_{k, l}-L, t\right) \geq \varepsilon \delta
\end{aligned}
$$

which implies

$$
\begin{aligned}
& \left\{(m, n) \in \mathbb{N} \times \mathbb{N}: \frac{1}{\lambda_{m n}^{\alpha}} \mid\left\{(k, l) \in I_{m n}: \mu\left(x_{k, l}-L, t\right) \leq 1-\varepsilon \text { or } v\left(x_{k, l}-L, t\right) \geq \varepsilon\right\} \mid \geq \delta\right\} \\
\subset \quad & \left\{(m, n) \in \mathbb{N} \times \mathbb{N}: \frac{1}{\lambda_{m n}^{\alpha}}\left\{\sum_{(k, l) \in I_{m n}} \mu\left(x_{k, l}-L, t\right) \leq 1-\varepsilon \text { or } \sum_{(k, l) \in I_{m n}} v\left(x_{k, l}-L, t\right) \geq \varepsilon\right\} \geq \varepsilon \delta\right\}
\end{aligned}
$$

Since $x_{k, l} \rightarrow L\left([V, \lambda]_{2}^{\alpha}(\mathcal{I})^{(\mu, v)}\right)$, we immediately see that $x_{k, l} \rightarrow L\left(S_{\lambda}^{\alpha}(\mathcal{I})_{2}^{(\mu, v)}\right)$, this completed the proof of the theorem.

Theorem 2. Let $(X, \mu, v, *, \diamond)$ be an IFNS. $S^{\alpha}(\mathcal{I})_{2}^{(\mu, v)} \subset S_{\lambda}^{\alpha}(\mathcal{I})_{2}^{(\mu, v)}$ if $\lim \inf _{m n \rightarrow \infty} \frac{\lambda_{m n}^{\alpha}}{(m n)^{\alpha}}>0$.

Proof. For given $\varepsilon>0$ and every $t>0$, we have

$$
\begin{aligned}
& \frac{1}{(m n)^{\alpha}} \mid\left\{k \leq m \text { and } l \leq n: \mu\left(x_{k, l}-L, t\right) \leq 1-\varepsilon \text { or } v\left(x_{k, l}-L, t\right) \geq \varepsilon\right\} \mid \\
\geq & \frac{1}{(m n)^{\alpha}} \mid\left\{(k, l) \in I_{m n}: \mu\left(x_{k, l}-L, t\right) \leq 1-\varepsilon \text { or } v\left(x_{k, l}-L, t\right) \geq \varepsilon\right\} \mid \\
= & \frac{\lambda_{m n}^{\alpha}}{(m n)^{\alpha}} \frac{1}{\lambda_{m n}^{\alpha}} \mid\left\{(k, l) \in I_{m n}: \mu\left(x_{k, l}-L, t\right) \leq 1-\varepsilon \text { or } v\left(x_{k, l}-L, t\right) \geq \varepsilon\right\} \mid
\end{aligned}
$$

If $\lim _{n \rightarrow \infty} \frac{\lambda_{m n}^{\alpha}}{(m n)^{\alpha}}=\alpha$ then from definition $\left\{(m, n) \in \mathbb{N} \times \mathbb{N}: \frac{\lambda_{m n}^{\alpha}}{(m n)^{\alpha}}<\frac{\alpha}{2}\right\}$ is finite. For every $\delta>0$,

$$
\begin{aligned}
& \left\{(m, n) \in \mathbb{N} \times \mathbb{N}: \frac{1}{\lambda_{m n}^{\alpha}} \mid\left\{(k, l) \in I_{m n}: \mu\left(x_{k, l}-L, t\right) \leq 1-\varepsilon \text { or } v\left(x_{k, l}-L, t\right) \geq \varepsilon\right\} \mid \geq \delta\right\} \\
\subset & \left\{(m, n) \in \mathbb{N} \times \mathbb{N}: \frac{1}{(m n)^{\alpha}} \mid\left\{(k, l) \in I_{m n}: \mu\left(x_{k, l}-L, t\right) \leq 1-\varepsilon \text { or } v\left(x_{k, l}-L, t\right) \geq \varepsilon\right\} \mid \geq \frac{\alpha}{2} \delta\right\} \\
& \cup\left\{(m, n) \in \mathbb{N} \times \mathbb{N}: \frac{\lambda_{m n}^{\alpha}}{(m n)^{\alpha}}<\frac{\alpha}{2}\right\}
\end{aligned}
$$

Since $\mathcal{I}$ is admissible, the set on the right-hand side belongs to $\mathcal{I}$ and this completed the proof of the theorem.

It is easy to check that both $S^{\alpha}(\mathcal{I})_{2}^{(\mu, v)}$ and $S_{\lambda}^{\alpha}(\mathcal{I})_{2}^{(\mu, v)}$ are linear subspaces of the space of real sequences. Next we present a topological characterization of these spaces. As both the proofs are similar, we give the detailed proof for the class $S_{\lambda}^{\alpha}(\mathcal{I})_{2}^{(\mu, v)}$ only. 
Theorem 3. Let $(X, \mu, v, *, \diamond)$ be an IFNS such that $\frac{\varepsilon_{m n}}{4} \oslash \frac{\varepsilon_{m n}}{4}<\frac{\varepsilon_{m n}}{2}$ and $\left(1-\frac{\varepsilon_{m n}}{4}\right) *\left(1-\frac{\varepsilon_{m n}}{4}\right)>1-\frac{\varepsilon_{m n}}{2}$. If $X$ is a Banach space then $S_{\lambda}^{\alpha}(\mathcal{I})_{2}^{(\mu, v)} \cap \ell_{\infty}^{(\mu, v)_{2}}$ is a closed subset of $\ell_{\infty}^{(\mu, v)}$, where $\ell_{\infty}^{(\mu, v)}$ stands for the space of all double bounded sequences of intuitionistic fuzzy norm $(\mu, v)$.

Proof. We first assume that $\left(x^{m n}\right) \subset S_{\lambda}^{\alpha}(\mathcal{I})_{2}^{(\mu, v)} \cap \ell_{\infty}^{(\mu, v)}, 0<\alpha \leq 1$, is a convergent sequence and it converges to $x \in \ell_{\infty}^{(\mu, v)}$. We need to show that $x \in S_{\lambda}^{\alpha}(\mathcal{I})^{(\mu, v)} \cap \ell_{\infty}^{(\mu, v)}$. Suppose that $x^{m n} \rightarrow L_{m n}\left(S_{\lambda}^{\alpha}(\mathcal{I})_{2}^{(\mu, v)}\right)$ for all $(m, n) \in \mathbb{N} \times \mathbb{N}$. Take a sequence $\left\{\varepsilon_{m n}\right\}$ of strictly decreasing positive numbers converging to zero. We can find an $(m, n) \in \mathbb{N} \times \mathbb{N}$ such that $\sup v\left(x-x^{m n}, t\right)<\frac{\varepsilon_{m n}}{4}$ for all $m \geq m_{0}$ and $n \geq n_{0}$.

Write

$$
A_{\mu, v}\left(\varepsilon_{m n}, t\right)=\left\{(m, n) \in \mathbb{N} \times \mathbb{N}: \frac{1}{\lambda_{m n}^{\alpha}}\left|\left\{\begin{array}{c}
(k, l) \in I_{m n}: \mu\left(x_{k, l}^{m n}-L_{m n}, t\right) \leq 1-\frac{\varepsilon_{m n}}{4} \text { or } \\
v\left(x_{k, l}^{m n}-L_{m n}, t\right) \geq \frac{\varepsilon_{m n}}{4}
\end{array}\right\}\right|<\delta\right\}
$$

belongs to $F(\mathcal{I})$ and

$$
B_{\mu, v}\left(\varepsilon_{n}, t\right)=\left\{(m, n) \in \mathbb{N} \times \mathbb{N}: \frac{1}{\lambda_{m n}^{\alpha}}\left|\left\{\begin{array}{c}
(k, l) \in I_{m n}: \mu\left(x_{k, l}^{m+1, n+1}-L_{m+1, n+1}, t\right) \leq 1-\frac{\varepsilon_{m n}}{4} \text { or } \\
v\left(x_{k, l}^{m+l, n+1}-L_{m+1, n+1}, t\right) \geq \frac{\varepsilon_{m n}}{4}
\end{array}\right\}\right|<\delta\right\}
$$

belongs to $F(\mathcal{I})$. Since $A_{\mu, v}\left(\varepsilon_{m n}, t\right) \cap B_{\mu, v}\left(\varepsilon_{m n}, t\right) \in F(\mathcal{I})$ and $\varnothing \notin F(\mathcal{I})$, we can choose $(m, n) \in$ $A_{\mu, v}\left(\varepsilon_{m n}, t\right) \cap B_{\mu, v}\left(\varepsilon_{m n}, t\right)$. Then

$$
\frac{1}{\lambda_{m n}^{\alpha}}\left|\left\{\begin{array}{c}
(k, l) \in I_{m n}: \mu\left(x_{k, l}^{m n}-L_{m n}, t\right) \leq 1-\frac{\varepsilon_{m n}}{4} \text { or } v\left(x_{k, l}^{m n}-L_{m n}, t\right) \geq \frac{\varepsilon_{m n}}{4} \vee \\
\mu\left(x_{k, l}^{m+1, n+1}-L_{m+1, n+1}, t\right) \leq 1-\frac{\varepsilon_{m n}}{4} \text { or } v\left(x_{k, l}^{m+1, n+1}-L_{m+1, n+1}, t\right) \geq \frac{\varepsilon_{m n}}{4}
\end{array}\right\}\right| \leq 2 \delta<1
$$

Since $\lambda_{m n} \rightarrow \infty$ and $A_{\mu, v}\left(\varepsilon_{m n}, t\right) \cap B_{\mu, v}\left(\varepsilon_{m n}, t\right) \in F(\mathcal{I})$ is infinite, we can actually choose the above $m, n$ so that $\lambda_{m m}>5$. Hence there must exist a $(k, l) \in I_{m n}$ for which we have simultaneously, $\mu\left(x_{k, l}^{m n}-L_{m n}, t\right)>1-\frac{\varepsilon_{m n}}{4}$ or $v\left(x_{k, l}^{m n}-L_{m n}, t\right)<\frac{\varepsilon_{m n}}{4}$ and $\mu\left(x_{k, l}^{m+1, n+1}-L_{m+1, n+1}, t\right)>1-\frac{\varepsilon_{m n}}{4}$ or $v\left(x_{k, l}^{m+1, n+1}-L_{m+1, n+1}, t\right)<\frac{\varepsilon_{m n}}{4}$. For a given $\varepsilon_{m n}>0$ choose $\frac{\varepsilon_{m n}}{2}$ such that $\left(1-\frac{\varepsilon_{m n}}{2}\right) *\left(1-\frac{\varepsilon_{m n}}{2}\right)>$ $1-\varepsilon_{m n}$ and $\frac{\varepsilon_{m n}}{2} \diamond \frac{\varepsilon_{m n}}{2}<\varepsilon_{n}$. Then it follows that

$$
v\left(L_{m n}-x_{k, l}^{m n}, \frac{t}{2}\right) \diamond v\left(L_{m+1, n+1}-x_{k, l}^{m+1, n+1}, \frac{t}{2}\right) \leq \frac{\varepsilon_{m n}}{4} \diamond \frac{\varepsilon_{m n}}{4}<\frac{\varepsilon_{m n}}{2}
$$

and

$$
\begin{aligned}
v\left(x_{k, l}^{m n}-x_{k, l}^{m+1, n+1}, t\right) & \leq \sup _{m n} v\left(x-x^{m n}, \frac{t}{2}\right) \diamond \sup _{m n} v\left(x-x^{m+1, n+1}, \frac{t}{2}\right) \\
& \leq \frac{\varepsilon_{m n}}{4} \diamond \frac{\varepsilon_{m n}}{4}<\frac{\varepsilon_{m n}}{2}
\end{aligned}
$$

Hence we have

$$
\begin{aligned}
v\left(L_{m n}-L_{m+1, n+1}, t\right) & \leq\left[v\left(L_{m n}-x_{k, l}^{m n}, \frac{t}{3}\right) \diamond v\left(x_{k, l}^{m+1, n+1}-L_{m+1, n+1}, \frac{t}{3}\right)\right] \diamond v\left(x_{k, l}^{m n}-x_{k, l}^{m+1, n+1}, \frac{t}{3}\right) \\
& \leq \frac{\varepsilon_{m n}}{2} \diamond \frac{\varepsilon_{m n}}{2}<\varepsilon_{m n}
\end{aligned}
$$

and similarly we have $\mu\left(L_{m n}-L_{m+1, n+1}, t\right)>1-\varepsilon_{m n}$. This implies that $\left\{L_{m n}\right\}$ is a Cauchy sequence in $X$ and let $L_{m n} \rightarrow L \in X$ as $m, n \rightarrow \infty$. We shall prove that $x \rightarrow L\left(S_{\lambda}^{\alpha}(\mathcal{I})_{2}^{(\mu, v)}\right)$. For any $\varepsilon>0$ and 
$t>0$, choose $(m, n) \in \mathbb{N} \times \mathbb{N}$ such that $\varepsilon_{m n}<\frac{\varepsilon}{4}, \sup _{m n} v\left(x-x_{k, l}^{m n}, t\right)<\frac{\varepsilon}{4}, \mu\left(L_{m n}-L, t\right)>1-\frac{\varepsilon}{4}$ or $v\left(L_{m n}-L, t\right)<\frac{\varepsilon}{4}$. Now since

$$
\begin{aligned}
& \frac{1}{\lambda_{m n}^{\alpha}}\left|\left\{(k, l) \in I_{m n}: v\left(x_{k, l}-L, t\right) \geq \varepsilon\right\}\right| \\
\leq & \frac{1}{\lambda_{m n}^{\alpha}}\left|\left\{(k, l) \in I_{m n}: v\left(x_{k, l}-x_{k, l}^{m n}, \frac{t}{3}\right) \diamond\left[v\left(x_{k, l}^{m n}-L_{m n}, \frac{t}{3}\right) \diamond v\left(L_{m n}-L, \frac{t}{3}\right)\right] \geq \varepsilon\right\}\right| \\
\leq & \frac{1}{\lambda_{m n}^{\alpha}}\left|\left\{(k, l) \in I_{m n}: v\left(x_{k, l}^{m n}-L_{m n}, \frac{t}{3}\right) \geq \frac{\varepsilon}{2}\right\}\right|
\end{aligned}
$$

and similarly

$$
\frac{1}{\lambda_{m n}^{\alpha}}\left|\left\{(k, l) \in I_{m n}: \mu\left(x_{k, l}-L, t\right) \leq 1-\varepsilon\right\}\right|>\frac{1}{\lambda_{m n}^{\alpha}}\left|\left\{(k, l) \in I_{m n}: \mu\left(x_{k, l}^{m n}-L, \frac{t}{3}\right) \leq 1-\frac{\varepsilon}{2}\right\}\right|
$$

it follows that

$$
\begin{aligned}
& \left\{(m, n) \in \mathbb{N} \times \mathbb{N}: \frac{1}{\lambda_{m n}^{\alpha}} \mid\left\{(k, l) \in I_{m n}: \mu\left(x_{k, l}^{m n}-L, t\right) \leq 1-\varepsilon \text { or } v\left(x_{k, l}^{m n}-L, t\right) \geq \varepsilon\right\} \mid \geq \delta\right\} \\
\subset \quad & \left\{(m, n) \in \mathbb{N} \times \mathbb{N}: \frac{1}{\lambda_{m n}^{\alpha}} \mid\left\{(k, l) \in I_{m n}: \mu\left(x_{k, l}^{m n}-L, \frac{t}{3}\right) \leq 1-\frac{\varepsilon}{2} \text { or } v\left(x_{k, l}^{m n}-L, \frac{t}{3}\right) \geq \frac{\varepsilon}{2}\right\} \mid \geq \delta\right\}
\end{aligned}
$$

for any given $\delta>0$. Hence we have $x \rightarrow L\left(S_{\lambda}^{\alpha}(\mathcal{I})_{2}^{(\mu, v)}\right)$. This completes the proof of the theorem.

\section{Conclusions}

We should note that the notions of $\mathcal{I}$-double statistical convergence and $\mathcal{I}_{\lambda}$-double statistical convergence of order $\alpha$ has not been studied in the setting of fuzzy normed linear spaces until now. For this reason, in this paper the notions of $\mathcal{I}$-double statistical convergence and $\mathcal{I}_{\lambda}$-double statistical convergence of order $\alpha$ are presented in an intuitionistic fuzzy normed linear space and some important results are given. We think that this method can also be implemented to other linear space.

Conflicts of Interest: The authors declare no conflict of interest.

\section{References}

1. Zadeh, L.A. Fuzzy sets. Inf. Control 1965, 8, 338-353.

2. Atanassov, K.T. Intuitionistic fuzzy sets. Fuzzy Sets Syst. 1986, 2, 87-96.

3. Park, J.H. Intuitionistic fuzzy metric spaces. Chaos Solitons Fractals 2004, 22, 1039-1046.

4. Saadati, R.; Park, J.H. On the intuitioistic fuzzy topologicial spaces. Chaos Solitons Fractals 2006, 27, 331-344.

5. El Naschie, M.S. On certainty of Cantorian geometry and two-slit experiment. Chaos Solitons Fractals 1998, 9 , 517-529.

6. Savaş, E. $\lambda$-double sequence spaces of fuzzy real numbers defined by Orlicz function. Math. Commun. 2009, 14, 287-297.

7. Fast, H. Sur la convergence statistique. Colloq. Math. 1951, 2, 241-244.

8. Schoenberg, I.J. The integrability of certain functions and related summability methods. Am. Math. Mon. 1959, 66, 361-375.

9. Fridy, J.A. On statistical convergence. Analysis 1985, 5, 301-313.

10. Šalát, T. On statistically convergent sequences of real numbers. Math. Slovaca 1980, 30, 139-150.

11. Cakalli, H. A study on statistical convergence. Funct. Anal. Approx. Comput. 2009, 1, 19-24.

12. Maio, G.D.; Kocinac, L.D.R. Statistical convergence in topology. Topol. Appl. 2008, 156, $28-45$.

13. Freedman, A.R.; Sember, J.J.; Raphael, M. Some Cesàro-type summability spaces. Proc. Lond. Math. Soc. 1978, 37, 508-520.

14. Mursaleen, M. $\lambda$-statistical convergence. Math. Slovaca 2000, 50, 111-115.

15. Savaş, E.; Das, P. A generalized statistical convergence via ideals. Appl. Math. Lett. 2011, 24, 826-830. 
16. Colak, R. Statistical Convergence of Order $\alpha$, Modern Methods in Analysis and Its Applications; Anamaya Pub.: New Delhi, India, 2010; pp. 121-129.

17. Colak, R.; Bektas, C.A. $\lambda$-statistical convergence of order $\alpha$. Acta Math. Sci. 2011, 31, 953-959.

18. Kostyrko, P.; Šalát, T.; Wilczynki, W. I-convergence. Real Anal. Exch. 2000-2001, 26, 669-685.

19. Das, P.; Savaş, E.; Ghosal, S.K. On generalizations of certain summability methods using ideals. Appl. Math. Lett. 2011, 24, 1509-1514.

20. Das, P.; Ghosal, S. Some further results on $\mathcal{I}$-Cauchy sequences and condition (AP). Comput. Math. Appl. 2010, 59, 2597-2600.

21. Savaş, E. $\triangle^{m}$-strongly summable sequences spaces in 2-Normed Spaces defined by Ideal Convergence and an Orlicz Function. Appl. Math. Comput. 2010, 217, 271-276.

22. Savaş, E.; Das, P.; Sudipta, D. A note on strong matrix summability via ideals. Appl. Math. Lett. 2012, 25, 733-738.

23. Savaş, E. Some $\mathcal{I}$-convergent sequence spaces of fuzzy numbers defined by infinite matrix. Math. Comput. Appl. 2013, 18, 84-93.

24. Savaş, E. On generalized double statistical convergence via ideals. In Proceedings of the Fifth Saudi Science Conference, Makkah, Saudi Arabia, 16-18 April 2012.

25. Savaş, E. On strong double matrix summability via ideals. Filomat 2012, 26, 1143-1150.

26. Mohiuddine, S.A.; Danish Lohani, Q.M. On generalized statistical convergence in intuitionistic fuzzy normed spaces. Chaos Solitons Fractals 2009, 42, 1731-1737.

27. Mursaleen, M.; Mohiuddine, S.A.; Edely, H.H. On the ideal convergence of double sequences in intuitionistic fuzzy normed spaces. Comput. Math. Appl. 2010, 59, 603-611.

28. Schweizer, B.; Sklar, A. Statistical metric spaces. Pac. J. Math. 1960, 10, 313-334.

29. Kumar, V.; Mursaleen, M. On $(\lambda, \mu)$-Statistical convergence of double sequences on intuitionistic fuzzy normed spaces. Filomat 2011, 25, 109-120.

30. Savaş, E. On strongly $\lambda$-summable sequences of fuzzy numbers. Inf. Sci. 2000, 125, 181-186.

31. Karakus, S.; Demirci, K.; Duman, O. Statistical convergence on intuitionistic fuzzy normed spaces. Chaos Solitons Fractals 2008, 35, 763-769.

32. Savas, E.; Mursaleen, M. On statistically convergent double sequence of Fuzzy numbers. Inf. Sci. 2004, 162, 183-192.

33. Mursaleen, M.; Edely, O.H. Statistical convergence of double sequences. J. Math. Anal. Appl. 2003, 288, 223-231.

34. Mursaleen, M.; Mohiuddine, S.A. Statistical convergence of double sequences in intuitionistic fuzzy normed spaces. Chaos Solitons Fractals 2009, 41, 2414-2421.

(C) 2016 by the author; licensee MDPI, Basel, Switzerland. This article is an open access article distributed under the terms and conditions of the Creative Commons Attribution (CC-BY) license (http:/ / creativecommons.org/licenses/by/4.0/). 